\title{
Atezolizumab in advanced non-small cell lung cancer
}

\author{
Marius Ilie ${ }^{1,2,3}$, Paul Hofman ${ }^{1,2,3}$ \\ ${ }^{1}$ Université Côte d'Azur, University Hospital Federation OncoAge, Laboratory of Clinical and Experimental Pathology, Hôpital Pasteur, Nice, \\ France; ${ }^{2}$ Université Côte d'Azur, Institute for Research on Cancer and Ageing, Nice (IR-CAN), Inserm U1081 and UMR CNRS 7284, Nice, \\ France; ${ }^{3}$ Université Côte d'Azur, University Hospital Federation OncoAge, Hospital-Related Biobank (BB-0033-00025), Hôpital Pasteur, Nice, \\ France \\ Correspondence to: Paul Hofman. Laboratory of Clinical and Experimental Pathology, Pasteur Hospital, 30 avenue de la Voie Romaine, 06002 Nice, \\ France. Email: hofman.p@chu-nice.fr. \\ Provenance: This is an invited Editorial commissioned by the Section Editor Ming-hui Zhang (Department of Medical Oncology, Harbin Medical \\ University Cancer Hospital, Harbin, China). \\ Comment on: Peters S, Gettinger S, Johnson ML, et al. Phase II Trial of Atezolizumab As First-Line or Subsequent Therapy for Patients With \\ Programmed Death-Ligand 1-Selected Advanced Non-Small-Cell Lung Cancer (BIRCH). J Clin Oncol 2017;35:2781-9.
}

Submitted Sep 02, 2017. Accepted for publication Sep 13, 2017.

doi: $10.21037 /$ jtd.2017.09.73

View this article at: http://dx.doi.org/10.21037/jtd.2017.09.73

Atezolizumab (Tecentriq ${ }^{\circledR}$, MPDL3280A, Genentech, CA, USA) is a humanized IgG1 antagonist antibody to PD-L1 engineered to avoid antibody-dependent cell-mediated cytotoxicity of activated T cells that may express PD-L1 (1). It blocks directly the interaction between the PD-L1 and PD-1/B7.1 receptors activation complex, hence it activates the tumor-specific immune response, and induces tumor cell killing (1).

In patients with non-small cell lung cancer (NSCLC) expressing PD-L1 atezolizumab can improve the proportion of patients achieving an objective response rate (ORR) compared with historical controls, according to the results of a new study (2). In the open-label phase II BIRCH trial of 659 patients whose tumors expressed PD-L1 on $\geq 5 \%$ PD-L1-expressing tumor cells (TC2) or tumor-infiltrating immune cells (IC2), using the SP142 immunohistochemistry (IHC) assay (Ventana Medical Systems, Tucson, AZ), ORR associated with atezolizumab (1200 mg IV every three weeks) were $22 \%$ among treatment-naïve patients, $19 \%$ among patients being treated in the second-line setting, and $18 \%$ among those receiving atezolizumab as third-line therapy. Higher ORR (26-31\%) was observed in the TC3 ( $\geq 50 \%$ TC) or IC3 ( $\geq 10 \%$ IC) subgroups for both first-line and $\geq$ second line, although in first-line patients it was comparable to chemotherapy. The epidermal growth factor receptor (EGFR) or KRAS mutation status had no impact on treatment responses. The efficacy of atezolizumab could not be assessed in patients with rearranged anaplastic lymphoma kinase (ALK) due to an insufficient number of patients. An updated survival analysis at 20 months of follow-up showed that the median overall survival (OS) was highest in first-line treated patients at 23.5 months (95\% CI: 18.1 months-not estimable) vs. 15.5 (12.3-19.3) months in the second-line cohort and 13.2 (10.3-17.5) months in the third-line cohort. Unlike the ORR, the PD-L1 status seemed to have no impact on OS. Nonetheless, the treatment with atezolizumab improved the OS relative to platinum-based chemotherapy (23.5 vs. 10 to 12 months) for patients who received first-line treatment and showing high PD-L1 expression (e.g., TC2/3 or IC2/3 patients and TC3 or IC3 subgroup) (2).

Similar results were reported in the POPLAR and OAK trials, in which the ORR and median PFS results underestimated the broad OS benefit seen with atezolizumab $v s$. docetaxel $(3,4)$. In these studies, the PD-L1 was predictive for clinical efficacy such as ORR, but may be less important for OS.

Overall, results from the BIRCH study demonstrated a clinically meaningful efficacy and safety of atezolizumab in all lines of therapy.

The efficacy of atezolizumab across these studies in terms of both efficacy as well as toxicity profiles over conventional chemotherapy has made it the third PD-1/ PD-L1 checkpoint inhibitor approved by the FDA for 
relapsed advanced NSCLC (5).

This study confirms that a PD-L1-selected group has a favorable outcome with agents that inhibit the interaction between PD-1 and PD-L1. However, while these results add to the evidence of a subgroup benefit with PD-L1 inhibitors, it does not have the power of a randomized controlled trial as the patients are compared to historical controls (6). Further studies are needed prior to use of atezolizumab in the first-line setting.

Atezolizumab represents a step forward in the treatment of patients with relapsed advanced NSCLC. Nonetheless, the choice of the most appropriate checkpoint inhibitor in the second-line setting between the three FDAapproved for use in NSCLC-atezolizumab, nivolumab, and pembrolizumab-is still unclear (5). All three are effective in second-line setting, with pembrolizumab being approved for use in NSCLC patients with $\geq 1 \%$ PD-L1 expression on tumor cells, while atezolizumab and nivolumab demonstrated clinical benefit over chemotherapy irrespective of the PD-L1 expression (7). Pembrolizumab is the only drug holding a first-line indication for singleagent treatment in NSCLC patients with $\geq 50 \%$ PD-L1 expression on tumor cells (8). The OAK study demonstrated clinical benefit over conventional chemotherapy in both squamous and non-squamous NSCLC, regardless of the PD-L1 expression. However, significant improvement of ORR and OS was observed in patients showing high PD-L1 expression, confirming the SP142 assay as a reliable predictive biomarker to select patients most likely to benefit from treatment with atezolizumab (4). Additional studies are needed to define further the place of these agents in the second-line setting.

Importantly, these studies also demonstrated that only optimal patient selection is the key to successful PD-1/PD-L1 inhibition. Efforts to standardize the different PD-L1 IHC assays and to identify additional predictive biomarkers are currently in progress (9).

At present, each immune checkpoint inhibitor was approved with its own companion or complementary PD-L1 expression assay. Thus, there is growing interest in assessing the interchangeability across different IHC assays. By opposition to companion tests, complementary assays do not restrict patients from receiving treatments based on the test result. This is because the biomarker status assessed by the complementary assay was not related to therapeutic benefit in all patients. Nevertheless, the biomarker status may inform on increased therapeutic benefits in some subgroups of patients. Currently, there are two PD-L1 complementary diagnostics, namely, the SP142 assay (Ventana) for use with atezolizumab (for bladder and NSCLC indications) and the 28-8 assay (Dako) for use with nivolumab (for melanoma and NSCLC indications) (10).

The PD-L1 SP142 IHC assay used in BIRCH, was specifically designed and validated for use with atezolizumab. Compared with the other commercial PD-L1 IHC assays (Dako 28-8, Dako 22C3 and Ventana SP263), the SP142 scoring is distinctive by the assessment of PD-L1 expression on both tumor cells and tumor-infiltrating immune cells (11-13). Recent standardization work demonstrated that the SP142 clone exhibited less overall stained tumor cells when compared to the other three IHC assays (11-13). Moreover, the SP142 assay showed the highest inter-pathologist variability, in particular when immune cells were scored (14). These results suggest, at least at this stage, that it is crucial to use the drug's companion/complementary IHC test with validated cutoff for PD-L1 positivity, in order to confidently obtain a clinical benefit specific to that particular treatment (15). Moreover, the IHC technique holds the intrinsic disadvantage of subjective interpretation, in particular when PD-L1 is expressed on both tumor cells as well as on tumorinfiltrating immune cells. Therefore, there is also growing interest in developing alternative methods for more reliable PD-L1 quantification. It is certainly possible that observer bias can lead to a shift towards the utilization of nivolumab, which does not require PD-L1 testing, and thus places extra emphasis on reducing inter-pathologist variability (15).

It would be of interest to evaluate tissue IHC assays in combination with multicolor flow cytometry to better characterize the immune tumor microenvironment by staining cells such as CD8+/CD4+ T cells, macrophages, myeloid-derived suppressor cells, natural-killers or regulatory $\mathrm{T}$ cells (16). These new methodologies may contribute to better biomarker assessment on clinical samples, as well as improve the predictive value of PD-L1 expression on both tumor cells and immune cells for immunotherapy.

Finally, some NSCLC patients without PD-L1 expression may still respond to checkpoint inhibitor treatment (9). Moreover, de novo resistance to immunotherapy has been reported in a relatively significant proportion of NSCLC patients, despite PD-L1 being expressed in tumors (9). Importantly, the intratumoral heterogeneity of PD-L1 expression may fail to appreciate the PD-L1 status in the whole tumor burden when assessed on biopsy samples (17). In addition, several blood-based assays are currently under investigation as predictive biomarkers for response to 
checkpoint inhibitors $(18,19)$.

In conclusion, future challenges will be to identify the most effective treatment sequence including immunotherapies, to standardize the different PD-L1 IHC assays, to understand the resistance mechanisms of checkpoint inhibitors, and to identify additional biomarkers to optimize benefit of immunotherapy for NSCLC patients.

\section{Acknowledgements}

The authors would like to thank "Conseil Départemental Alpes Maritimes 06", "Comité Départemental 06 de la Ligue contre le Cancer", and the "Cancéropôle PACA" for their financial support.

\section{Footnote}

Conflicts of Interest: Paul Hofman is a member of different industrial scientific advisory boards (Roche, AstraZeneca, Novartis, Bristol-Myers Squibb, Pfizer, Qiagen, Janssen, Biocartis) for which he receives honorarium. Marius Ilié has no conflicts of interest to declare.

\section{References}

1. Herbst RS, Soria JC, Kowanetz M, et al. Predictive correlates of response to the anti-PD-L1 antibody MPDL3280A in cancer patients. Nature 2014;515:563-7.

2. Peters $\mathrm{S}$, Gettinger S, Johnson ML, et al. Phase II Trial of Atezolizumab As First-Line or Subsequent Therapy for Patients With Programmed Death-Ligand 1-Selected Advanced Non-Small-Cell Lung Cancer (BIRCH). J Clin Oncol 2017;35:2781-9.

3. Fehrenbacher L, Spira A, Ballinger M, et al. Atezolizumab versus docetaxel for patients with previously treated non-small-cell lung cancer (POPLAR): a multicentre, open-label, phase 2 randomised controlled trial. Lancet 2016;387:1837-46.

4. Rittmeyer A, Barlesi F, Waterkamp D, et al. Atezolizumab versus docetaxel in patients with previously treated non-small-cell lung cancer (OAK): a phase 3, openlabel, multicentre randomised controlled trial. Lancet 2017;389:255-65.

5. Hanna N, Johnson D, Temin S, et al. Systemic Therapy for Stage IV Non-Small-Cell Lung Cancer: American Society of Clinical Oncology Clinical Practice Guideline Update. J Clin Oncol 2017;JCO2017746065.

6. Brower V. Atezolizumab in advanced non-small-cell lung cancer. Lancet Oncol 2017;18:e434.

7. Herbst RS, Baas P, Kim DW, et al. Pembrolizumab versus docetaxel for previously treated, PD-L1-positive, advanced non-small-cell lung cancer (KEYNOTE-010): a randomised controlled trial. Lancet 2016;387:1540-50.

8. Reck M, Rodriguez-Abreu D, Robinson AG, et al. Pembrolizumab versus Chemotherapy for PD-L1Positive Non-Small-Cell Lung Cancer. N Engl J Med 2016;375:1823-33.

9. Ilie M, Hofman V, Dietel M, et al. Assessment of the PD-L1 status by immunohistochemistry: challenges and perspectives for therapeutic strategies in lung cancer patients. Virchows Arch 2016;468:511-25.

10. Scheerens H, Malong A, Bassett K, et al. Current Status of Companion and Complementary Diagnostics: Strategic Considerations for Development and Launch. Clin Transl Sci 2017;10:84-92.

11. Rimm DL, Han G, Taube JM, et al. A Prospective, Multi-institutional, Pathologist-Based Assessment of 4 Immunohistochemistry Assays for PD-L1 Expression in Non-Small Cell Lung Cancer. JAMA Oncol 2017;3:1051-8.

12. Ratcliffe MJ, Sharpe A, Midha A, et al. Agreement between Programmed Cell Death Ligand-1 Diagnostic Assays across Multiple Protein Expression Cutoffs in Non-Small Cell Lung Cancer. Clin Cancer Res 2017;23:3585-91.

13. Hirsch FR, McElhinny A, Stanforth D, et al. PDL1 Immunohistochemistry Assays for Lung Cancer:

Results from Phase 1 of the Blueprint PD-L1 IHC Assay Comparison Project. J Thorac Oncol 2017;12:208-22.

14. Brunnstrom H, Johansson A, Westbom-Fremer S, et al. PD-L1 immunohistochemistry in clinical diagnostics of lung cancer: inter-pathologist variability is higher than assay variability. Mod Pathol 2017. [Epub ahead of print].

15. Chow JCH, Cheung KM, Cho WCS. Atezolizumab in non-small cell lung cancer: the era of precision immunooncology. Ann Transl Med 2017;5:265.

16. Chargin A, Morgan R, Sundram U, et al. Quantification of PD-L1 and PD-1 expression on tumor and immune cells in non-small cell lung cancer (NSCLC) using nonenzymatic tissue dissociation and flow cytometry. Cancer Immunol Immunother 2016;65:1317-23.

17. Ilie $\mathrm{M}$, Long-Mira $\mathrm{E}$, Bence $\mathrm{C}$, et al. Comparative study of the PD-L1 status between surgically resected specimens and matched biopsies of NSCLC patients reveal major discord-ances: a potential issue for anti-PD-L1 therapeutic 
strategies. Ann Oncol 2016;27:147-53.

18. Sanmamed MF, Perez-Gracia JL, Schalper KA, et al. Changes in serum interleukin-8 (IL-8) levels reflect and predict response to anti-PD-1 treatment in melanoma and non-small-cell lung cancer patients. Ann Oncol

Cite this article as: Ilie M, Hofman P. Atezolizumab in advanced non-small cell lung cancer. J Thorac Dis 2017;9(10):3603-3606. doi:10.21037/jtd.2017.09.73
2017;28:1988-95.

19. Strati A, Koutsodontis G, Papaxoinis G, et al. Prognostic significance of PD-L1 expression on circulating tumor cells in patients with head and neck squamous cell carcinoma. Ann Oncol 2017;28:1923-33. 